FILOLOGÍA INGLESA 



\title{
CLAVES PARA COMPRENDER LA DESTREZA DE LA COMPRENSIÓN ORAL EN LENGUA EXTRANJERA
}

\author{
NOA TALAVÁN ZANÓN \\ UNED. Madrid \\ ntalavan@flog.uned.es
}

\section{RESUMEN}

La destreza de la comprensión oral fue durante mucho tiempo una destreza desatendida en términos de investigación y búsqueda de métodos didácticos efectivos. Uno de los motivos principales de este abandono es que la comprensión oral es un proceso mental científicamente intangible en el que intervienen multitud de factores. Con el fin de llegar a comprender mejor esta destreza receptiva, este artículo explica los factores que intervienen en el proceso constructivo, expone los aspectos más relevantes de su enseñanza-aprendizaje y, por último, describe los fundamentos de la evaluación de la misma.

PALABRAS CLAVE: comprensión oral, proceso constructivo, alumno, evaluación.

\section{ABSTRACT}

Within the field of foreign language acquisition, oral comprehension was for a long time a neglected skill, both in terms of research and education. One of the main reasons for this abandonment is the fact that oral comprehension constitutes a scientifically intangible mental process where a great amount of factors are involved. The goal of this paper is to achieve a better understanding of this receptive skill; to that end, the main elements that take part in the constructive process are explained, the most relevant aspects of the teaching-learning process are described, and the basis of its assessment are also detailed. 
KEY WORDS: oral comprehension, constructive process, student, assessment.

La destreza de la Comprensión Oral (en adelante, $\mathrm{CO}$ ) ha sido durante mucho tiempo una destreza desatendida en el ámbito de la enseñanza-aprendizaje de lenguas extranjeras (en adelante, L2), en base a que se presuponía que podría perfeccionarse al hilo de la mejora de las demás destrezas. El hecho de que sea una de las destrezas más difíciles de enseñar (por la necesidad de recursos necesarios y por la multitud de elementos diversos que intervienen en su adquisición) también ha influido en esta tendencia a dejarla de lado. Afortunadamente, esta situación ha mejorado considerablemente en tiempos recientes gracias a los trabajos de diversos autores (Brown, 1995; Buck, 2001; Rost, 2002) que han reivindicado su relevancia, analizado en profundidad sus componentes y propuesto estrategias diversas para su correcta adquisición y enseñanza. No se puede olvidar que la $\mathrm{CO}$ es uno de los pilares más importantes de la comunicación, además de ser una de las destrezas que los estudiantes de L2, en particular los hispanohablantes, suelen encontrar más difíciles de desarrollar. ${ }^{1}$

Definir la destreza de la $\mathrm{CO}$ en $\mathrm{L} 2$ no es tarea fácil porque se trata de describir un proceso mental científicamente intangible; por ello, se tiende a explicar por medio de metáforas o analogías. Además, dado que supone el modo en que el sujeto construye el significado global de un texto oral, se trata de un proceso subjetivo, que depende de múltiples factores relacionados con el sujeto que lo construye en el momento en el que se produce; depende, asimismo, del tipo de texto y de la situación en la que se inserta. Rost (2002) llega a afirmar que existen tantas definiciones de $\mathrm{CO}$ como tipos existen de esta destreza. Por ello, para poder comprenderla mejor, este autor proporciona cuatro categorías alrededor de las cuales se suelen situar todas las posibles definiciones: (1) receptiva (la recepción de las palabras concretas del hablante), (2) constructiva (la construcción y la representación del mensaje), (3) negociadora (la negociación del significado con el hablante y la respuesta) y (4) transformativa (la creación de significado a través de la participación, la imaginación y la empatía). En términos generales, se podría decir que la definición más completa es la constructiva porque incluye en cierto modo la receptiva, y porque sin ella la negociadora y la transformativa no pueden existir. En este sentido, una de las mejores definiciones generales de esta destreza podría ser: «listening means reframing the speaker's message in a way that's relevant to you» (Rost, 2002:2). En definitiva, lo que se pretende que consigan los alumnos para poder intervenir en intercambios comunicativos eficientes.

A continuación, se explican los factores que intervienen en este proceso constructivo. Más adelante, se exponen los aspectos más relevantes de su enseñanza-aprendizaje y, por último, se resumen aquéllos relacionados con la evaluación de la CO.

\section{FACTORES DEL PROCESO CONSTRUCTIVO}

La $\mathrm{CO}$ es un área muy compleja dentro de la didáctica de lenguas, dada la gran cantidad de elementos cognitivos, externos, subjetivos, etc. que intervienen en el proceso de la escucha, y la dificultad de su medición o evaluación objetiva. En este sentido, Ander-

\footnotetext{
${ }^{1}$ Entre las razones más relevantes que impiden a los alumnos comprender el inglés están las grandes diferencias a nivel fonético y prosódico que existen entre ambas lenguas, que bloquean su progreso en CO: los sistemas vocálicos son distintos (en inglés se diferencian las vocales largas y cortas y hay una clara tendencia a la diptongación), muchos sonidos consonánticos ingleses no existen en castellano, el volumen en inglés tiende a ser más bajo y el tono más alto, etc.
} 
son y Lynch (1988) advierten de la imposibilidad de que profesores e investigadores alcancen a dominar esta destreza y su docencia, dado que no es posible poseer (al menos por el momento) un conocimiento directo del proceso de CO en sí mismo. Según Lynch (1996), esta imposibilidad de observar el proceso interno de esta destreza acarrea dos desventajas básicas para la enseñanza-aprendizaje de la L2: la dificultad que tienen los profesores para juzgar el grado de comprensión real de los alumnos y la dificultad de los alumnos para conocer su grado de comprensión de modo individual o en relación con sus compañeros.

Dado que este artículo se centra especialmente en el proceso constructivo de la $\mathrm{CO}$ (y no en el receptivo, negociador ni transformativo propiamente dichos), a continuación se desarrollan los tres factores fundamentales que conforman dicho proceso: (1) los interlocutores, (2) el texto escuchado (o input) y (3) la situación.

\subsection{Los interlocutores}

A primera vista, parece que el papel principal en la conversación corresponde al hablante y que el papel del oyente es más bien pasivo (por ello, ha tendido a ignorarse), cuando, en realidad, el papel del oyente tiene una relevancia igual o mayor, si cabe. Como bien apunta Rost (2002:50), «the listener contributes to the conversation and achieves meaning, and at times «clarifies» or even creates meaning in the speaker». Por este motivo, el análisis del discurso oral ha de entenderse siempre como un proceso en dos direcciones en el que ambas partes (hablante y oyente) dependen la una de la otra y contribuyen recíprocamente a crear el acto comunicativo.

Lynch (1996) distingue dos tipos de situaciones de CO según el papel de los interlocutores: en un sentido o en dos sentidos, según intervengan o no hablante y oyente al mismo tiempo. En los discursos en dos sentidos, el oyente tiene la oportunidad de pedir aclaraciones cuando lo estima necesario. Cuando la situación es en un sentido, el oyente no tiene la posibilidad de intervenir o responder en modo alguno, por lo que el papel que adopta es de oyente furtivo o externo.

En lo que respecta al hablante en concreto, éste ha de decidir cómo ordenar y «empaquetar» la información de modo que el oyente pueda comprenderla dentro del contexto social y comunicativo en el que tiene lugar la interacción (Brown, 1995). Normalmente, en las clases de L2, en muchas de las tareas de $\mathrm{CO}$ (por ejemplo, escuchar un fragmento de audio y contestar preguntas léxicas o de contenido al respecto), no existe esta interacción entre el hablante y el oyente/alumno, ya que este último no suele participar en la situación comunicativa. Afortunadamente, en entornos didácticos más actuales, cada vez resulta más común encontrarse con otro tipo de situaciones comunicativas en las que el oyente/alumno sí interactúa con un hablante, que puede tomar la forma de profesor, compañero, texto procedente de tecnologías de la información y la comunicación (en adelante, TIC), etc.

Existen seis variables relativas al hablante que pueden facilitar o dificultar la comprensión por parte del oyente y que han de ser tenidas en cuenta (Weir, 2005): (1) la velocidad del discurso (cuanto más rápido, más complicado de comprender); (2) la variedad de acento (cuanto más fuerte, regional o desconocido sea el acento, más complicada será la comprensión); (3) la familiaridad de la voz del hablante (por ejemplo, los alumnos comprenden mejor al profesor, al estar acostumbrados a su voz, que a hablantes con vo- 
ces que nunca antes han oído); (4) el número de hablantes (a menor número, mayor facilidad); (5) la distinción entre ellos (por ejemplo, una situación con muchos hablantes masculinos será más difícil de comprender que una situación con menos hablantes y de distinto sexo); (6) y el sexo (si el sexo del hablante es el mismo que el del oyente o si el oyente está más acostumbrado a interactuar con personas de ese sexo, la comprensión también será más fácil).

En lo que al oyente se refiere, se pueden distinguir seis características fundamentales que ejercen una influencia importante en su actividad de CO (Cornaire, 1998): (1) la competencia lingüística, (2) la memoria a corto plazo, (3) el grado de atención, (4) la afectividad, (5) la edad y (6) los conocimientos (contextuales) anteriores.

1. En lo que respecta a la competencia lingüística, es necesario diferenciar entre la capacidad de reconocimiento y la capacidad de producción lingüística del interlocutor. Por otro lado, también se pueden distinguir distintos niveles de competencia a la hora de poner en práctica esta destreza: morfológico, léxico, fonético, prosódico, utilización de reglas gramaticales, capacidad de integrar rasgos no verbales, etc.

2. Aunque la distinción cognitiva tradicional de los tipos de memoria incluye tanto la memoria a largo plazo como la memoria a corto plazo, en el proceso de $\mathrm{CO}$, el interés se centra en esta última (Rost, 2002). Esto es así porque lo que se necesita para comprender un texto oral es el tipo de conocimiento que se activa en un momento determinado y que ayuda a comprender el texto escuchado a tiempo real. Obviamente, la información conocida y almacenada en la memoria a largo plazo también juega su papel en este proceso.

3. La falta de atención puede originar serios problemas para la CO. Los estudiantes más capaces son conscientes de las posibles faltas de atención y procuran compensarlas. Sin embargo, existen personas con problemas de concentración mayores que, cuando encuentran una dificultad, simplemente dejan de escuchar (Cornaire, 1998). Según Cohen (1990:42), «it appears that, on the average, 50 per cent of students are attending to the content of a lesson at a given moment, and most are just repeating the material to themselves (rather than performing any higher functions on the material)». Para Rost (2002), estas situaciones de falta de atención tienen lugar porque la atención es un sistema de capacidad limitada; por este motivo, este autor recomienda aprender a utilizar la atención selectiva. Este tipo de atención permite a los aprendices centrarse en lo que están escuchando, anulando cualquier otro detalle que pueda servir de distracción. Para conseguir desarrollar esta atención selectiva, se pueden utilizar diversas estrategias (como el uso de vídeo, imágenes, asociaciones, imaginación, etc). En palabras de Cohen (1990:43):

The question of concern to the language learner which emerges from a discussion of attention is: «Am I attending in class at a level that will ensure that language learning takes place?» For maximal learning to go on, you would be directing primary attention to the input, namely, the language instruction-such as the teacher's presentations, explanations, gestures, and words written on the board or in handouts; or another student's question and the teacher's answer. 
Prestar la atención adecuada al input es, pues, un elemento fundamental para que se produzca tanto la comprensión como el aprendizaje deseado.

4. La afectividad, ya sea en términos de actitud o motivación, es también un factor esencial. A partir de la importancia otorgada al llamado «filtro afectivo» en el Enfoque Natural (Krashen y Terrell, 1983), el elemento de la afectividad se ha considerdo con atención dentro del proceso de aprendizaje. Entre los diversos factores afectivos que pueden influir de modo positivo o negativo en el desarrollo de la CO, se pueden distinguir: la confianza en uno mismo, las creencias, los sentimientos y las actitudes de profesor y alumno, la afinidad hacia una lengua, la influencia de dicha lengua en la lengua materna, etc. Desgraciadamente, los efectos concretos de los factores de actitud y motivación son difíciles de investigar de un modo sistemático, debido a su gran subjetividad y a las diferencias que existen entre individuos (De Bot et al., 2005).

5. La edad, por su parte, afecta en gran medida al desarrollo de esta destreza, y prueba de ello es la gran cantidad de estudios que versan sobre las diferencias entre la forma y la capacidad de aprendizaje de niños y adultos, e incluso entre adultos y adolescentes (Cornaire, 1998; De Bot et al., 2005).

6. En lo que respecta a la relevancia de los conocimientos anteriores o previos, estos son muy importantes en el proceso constructivo de la CO. Son los cimientos a partir de los cuales se puede construir el mensaje y crear nuevos conocimientos. Conviene señalar los tipos de conocimiento utilizados para comprender lo que se escucha: los modelos más conocidos son los llamados procesos de arriba abajo (top-down $)^{2}$ y de abajo arriba (bottom-up $)^{3}$, que se refieren al orden en el que los distintos conocimientos se aplican para comprender un texto.

La CO tiende a ser un proceso de arriba abajo, ya que para comprender algo hay que hacer uso de diversos tipos de conocimiento que interactúan y que, además, no se aplican en un orden fijo (Buck, 2001). Esto no quiere decir que haya que olvidar los procesos de abajo arriba, puesto que la comprensión detallada de la información linguíística propiamente dicha sigue este modelo y es una de las fuentes de conocimiento que influyen en la descodificación inicial del texto.

El análisis precedente muestra la gran importancia del oyente en la comunicación. Él decide y elige qué aspectos del mensaje deben recibir prioridad, mientras que el hablante se comunica con el objetivo principal de ser escuchado por él. No obstante, el oyente no se define solamente por su papel en la interacción, como se comenta en la siguiente cita:

\footnotetext{
${ }^{2}$ Un proceso de $\mathrm{CO}$ de arriba abajo asume que el oyente construye o reconstruye de forma activa el significado original emitido por el hablante, partiendo de los conceptos más generales para ir «bajando» hacia los aspectos más particulares del texto; para ello, usa el conocimiento previo del contexto y de la situación de la escucha.

${ }^{3}$ El modelo de abajo arriba va de lo particular a lo más general del texto. Considera la CO como una descodificación de sonidos que se escuchan de un modo lineal, desde las unidades más pequeñas hasta los textos más completos: los fonemas se unen para formar palabras, las palabras para formar sintagmas, los sintagmas, oraciones y las oraciones, textos completos (Nunan, 1999).
} 
L'auditeur n'est pas uniquement défini par son rôle dans l'interaction. C'est un individu psicologiquement, socialement et culturellement déterminé. Ses caractéristiques au moment où il se comporte en auditeur (certaines sont permanentes, d'autres momentanées) vont influer sur la participation à la communication (Gremmo y Holec, 1990:34).

Todos estos factores juntos, unidos a los elementos ya comentados de competencia lingüística, memoria a corto plazo, atención, afectividad, edad y conocimientos anteriores, influirán tanto en su grado de $\mathrm{CO}$ como en su colaboración dentro de la interacción comunicativa que tenga lugar en cada momento.

\subsection{El texto}

El texto oral (el input o información de entrada recibida) se diferencia en gran medida del texto escrito. De hecho, hay una serie de aspectos que son únicos del texto oral (Rost, 2002:171-2); entre ellos, destacan rasgos físicos (como la velocidad de habla, los sonidos de fondo, la entonación o las dudas), rasgos lingüísticos (como las expresiones coloquiales, el uso frecuente de la elipsis o los falsos comienzos) y rasgos psicológicos (como la posibilidad de interactuar con el hablante, de negociar con él para aclarar significados o de construir los mensajes a partir del contexto).

No obstante, la diferencia más evidente entre textos orales y escritos, y una de las más importantes, es que las personas normalmente no hablan con frases, sino con expresiones o cláusulas cortas, que se pueden denominar idea units o unidades de significado (Buck, 2001:9). Estas idea units están conectadas entre sí, más por factores de coherencia que por estructuras gramaticales concretas. En palabras de Halliday (1989:92): «writing creates a world of things; talking creates a world of happening»; por regla general, se habla con ideas acerca de hechos acontecidos, mientras que se escribe acerca de cosas concretas.

En lo que respecta a los tipos posibles de textos orales, conviene hacer una distinción entre discurso «planeado» y discurso «espontáneo» (Buck, 2001). Cabe señalar que el uso de textos orales espontáneos o auténticos para el desarrollo de la CO en L2 no ha estado siempre presente en las programaciones. En su lugar, la tendencia más generalizada durante décadas ha sido la utilización de textos «planeados», específicamente creados para su utilización en contextos educativos, manipulados en términos de nivel y énfasis lingüístico, con una velocidad de habla relativamente más lenta, y generalmente carentes de errores, falsos comienzos, interrupciones u otros rasgos comunes de la lengua hablada real. Cabe señalar que también podemos encontrar textos «planeados» que no son específicamente educativos, como los discursos o conferencias de profesores, políticos, expertos, etc.

La utilización de textos planeados en lugar de textos de discurso espontáneo puede acarrear problemas de comunicación, así como un cierto grado de decepción y frustración en los alumnos cuando éstos se enfrentan a conversaciones reales, tanto en calidad de espectadores como de participantes activos. Por este motivo, la utilización de materiales auténticos (como los clips de vídeo) se está fomentando cada vez más desde el comienzo del proceso de aprendizaje, con un matiz de fondo claramente constructivista. En palabras de Miller (2003:16), «the aim of all listening lessons 
should be to allow learners a greater degree of independence when confronted with listening to the foreign language in a real world context, and that means using authentic texts».

Los documentos auténticos deberían usarse con todos los niveles, desde el comienzo del aprendizaje. No obstante, su utilización con principiantes ha sido y es objeto de controversia: las opiniones se suelen dividir entre los partidarios de los documentos simplificados y los que defienden el uso de los documentos auténticos de carácter informativo. Trabajos como el de Herron (1994) muestran los beneficios que se derivan de la utilización de documentos auténticos con este tipo de alumnos; otros ejemplos de autores que también recomiendan esta práctica son Long (1990) y Bacon (1992). Al fin y al cabo, se trata de un procedimiento que reproduce la situación de inmersión lingüística (en la comunidad nativa), donde la mayor parte del lenguaje al que acceden los aprendices no está adaptado ni simplificado.

En segundo lugar, además del tipo de texto, es importante tener en cuenta el nivel de dificultad y la longitud. Aparentemente, a partir de los tres minutos de escucha, los esfuerzos por mantener la atención tienden a sobrecargar la memoria a corto plazo (Cornaire, 1998); por tanto, la longitud del texto parece influir en el nivel de dificultad. Por este motivo, se recomienda no sobrepasar esa duración textual (tres minutos) cuando se realice cualquier actividad de CO. Aparte de la longitud, las siguientes seis características se deben tener en cuenta para conseguir que un texto oral sea fácil de comprender (Brown, 1995): (1) el número de personajes u objetos ha de ser limitado; (2) han de distinguirse con claridad; (3) las relaciones espaciales han de ser simples; (4) el orden cronológico de los acontecimientos debe respetarse; (5) debe haber un vínculo que explique la unión entre los distintos enunciados (por ejemplo, causa-efecto); y (6) ha de existir la posibilidad de vincular fácilmente la información nueva con los conocimientos anteriores. Otros aspectos a considerar en la elección del texto porque pueden afectar directamente a su dificultad son: (1) la velocidad con la que hablan los interlocutores y sus acentos y entonación; (2) la complejidad del contenido en términos lingüísticos (gramática, vocabulario, etc.) y de conocimientos previos necesarios; (3) las pausas existentes entre unidades de significado o idea units (cuanto más largas, menor dificultad); y (4) la cantidad de apoyo extra proporcionado en forma de imágenes, esquemas, redundancias o apoyos visuales. Si estos u otros factores de dificultad semejantes no se tienen en cuenta en la elección del input, muchas tareas de CO pueden resultar inútiles para los alumnos, o puede que no se aprovechen como debieran todos los elementos relevantes del texto para desarrollar habilidades de $\mathrm{CO}$ (Buck, 2001).

Por último, la dificultad del texto radica también, en muchas ocasiones, en el tipo de contenido, es decir, en la naturaleza de la información del texto. El contenido puede ser abstracto (por ejemplo, acerca de la libertad o sobre temas filosóficos) o concreto (por ejemplo, acerca de una entrevista o la venta de una casa). Weir (2005:74) señala que, aunque ambos tipos de información pueden estar presentes en el mismo texto, el contenido abstracto puede aumentar la dificultad de procesamiento del texto, dado que «[it] may in itself be cognitively as well as linguistically more complex and more difficult to process». Al aumentar la abstracción, crece el esfuerzo necesario para procesar el mensaje, tanto cognitiva como lingüísticamente. 
Sin texto no hay comunicación, ya que este elemento es el que lleva consigo el mensaje y el que pone en contacto a los interlocutores. Aunque también existe la comunicación no verbal, en el ámbito de la $\mathrm{CO}$ el texto podría considerarse el punto de partida desde el cual se pueden desarrollar tareas diversas y poner en práctica las estrategias de comprensión necesarias en cada momento.

\subsection{La situación}

El tercer y último factor del proceso constructivo de la $\mathrm{CO}$ es la situación comunicativa. Dada la gran amplitud de contextos y factores que se incluyen en el concepto de situación, este apartado se centra en los siguientes aspectos, que se cuentan entre los más significativos: el contexto cultural, el contexto pragmático, el contexto cognitivo y el contexto situacional.

Primeramente, la comprensión de todo tipo de rasgos socio-culturales pertenecientes al contexto cultural es de primordial importancia para procesar bien una lengua y poder comunicarse de modo eficiente, ya que dichos rasgos habitan y se transmiten a través de la lengua. Desgraciadamente, tal y como expone Lado (1957:114), resulta difícil realizar transferencias entre culturas: «the patternings that make it possible for unique occurrences to operate as sames among the members of a culture did not develop for operation across cultures. When they do occur in contact across cultures, many instances of misinterpretation take place». En otras palabras, dado que resulta complicado transferir los rasgos-culturales de una lengua a otra (porque tienden a estar ligados inseparablemente a la lengua a la que pertenecen), es necesario conocer el contexto cultural de la L2 en sí mismo. La influencia de este contexto hace que la competencia comunicativa vaya mucho más allá de la habilidad de comprender oraciones gramaticalmente correctas. Significa conocer lo que resulta apropiado decir dependiendo del interlocutor y de la situación, así como comprender las creencias y los valores que están representados en las diversas formas y usos de la lengua (Peterson y Coltrane, 2003).

En segundo lugar, el elemento fundamental dentro del contexto pragmático es el concepto de las intenciones comunicativas. En este ámbito, entran en juego dos factores fundamentales que forman parte de la interpretación del mensaje: los actos de habla y el principio de cooperación.

La teoría de los actos de habla (Austin, 1962; Searle, 1969) explica que la comunicación es una negociación de significados donde la unidad mínima son los actos de habla; el acto de habla es, pues, la unidad comunicativa básica por medio de la cual se realiza una acción. Esta teoría enfatiza la importancia del contexto para la correcta interpretación por parte del oyente de las intenciones del hablante (como pueden ser ofrecer, saludar, prometer o avisar). Las distintas partes de un acto de habla son: la locución (las palabras enunciadas), la ilocución (lo que el hablante pretende hacer con ellas) y la perlocución (el efecto que esta ilocución tiene en el oyente). La segunda (ilocución) se ha de tener en cuenta en el proceso constructivo de la $\mathrm{CO}$, cuando el oyente trata de procesar el mensaje que el hablante pretende transmitir por medio de las palabras (locución) que utiliza. La perlocución, por último, entra dentro del aspecto negociador y transformativo de la $\mathrm{CO}$, y está directa- 
mente relacionada con la interacción y colaboración directa entre los interlocutores para crear significado.

El principio de cooperación fue descrito por Grice (1969), y se considera en pragmática el principio regulador de las relaciones interlingüísticas. Por medio de este principio, se explica la posibilidad de realizar actos de habla indirectos. Esto ocurre cuando no se respeta alguna de las siguientes cuatro normas, denominadas máximas de la conversación: (1) máxima de cantidad (el acto de habla ha de ser lo suficientemente informativo), (2) máxima de calidad (no se deben decir falsedades), (3) máxima de relación (sólo se ha de dar información relevante para la situación) y (4) máxima de modo (se ha de evitar la ambigüedad en la expresión). En condiciones normales, los participantes de una conversación tendrían que respetar dichas máximas conversacionales a fin de que se cumpla el principio de cooperación y la conversación fluya sin problemas (Rost, 1990). Cuando se infringe o se elude alguna de estas máximas en un acto de habla, éste se vuelve indirecto, y el oyente ha de interpretar o inferir, por medio de factores contextuales, la intención real del hablante. La diferencia entre el acto de habla directo y lo que significa en realidad tras infringir alguna máxima (lo que el oyente ha de inferir) constituye una implicatura conversacional.

En tercer lugar, en lo que respecta al contexto cognitivo, cabe destacar que el entorno cognitivo de cada interlocutor influye de modo relevante en el procesamiento de la información. Sperber y Wilson (1986) explican que, como oyentes, tendemos a interpretar lo que escuchamos mediante inferencias que tienen en cuenta los aspectos más relevantes o conocidos del contexto. La complicación estriba en que la base de dicha inferencia suele ser lo que el oyente entiende o interpreta del texto. Por esta razón, «there is a crucial relationship in real-life communication between the utterance and the context in which it occurs and what participants are prepared to imagine, or to infer from their previous experience» (Brown: 1995:13). Así, uno de los aspectos que influye de modo más significativo en la $\mathrm{CO}$ está en la mente del oyente, es absolutamente subjetivo y depende de múltiples variables individuales de todo orden.

Por último, uno de los principales problemas de comprensión posibles surge cuando una situación dada (el contexto situacional) influye de manera especial en la interpretación de los enunciados lingüísticos; de hecho, existen muchas frases y/o expresiones que cambian de significado dependiendo de la situación (Brown, 1995). Es necesario que el alumno esté preparado para todo tipo de situaciones porque, como explican Gremmo y Holec (1990:37): «progresser en compréhension orale, c'est savoir utiliser les connaisances dans des situations de compréhension de plus en plus variées: c'est ainsi qu' on peut mettre en oeuvre et developer des savoir-faire».

En los sentidos descritos se comprende cómo resulta absolutamente fundamental que el papel de la situación se tenga en cuenta en todo momento dentro de la enseñanza-aprendizaje de la CO en L2. Por último, no se puede olvidar que la situación también contiene factores tales como el espacio o el tiempo, las relaciones existentes entre los interlocutores, los conocimientos anteriores que comparten y los elementos no verbales. 


\section{EL PROCESO DE ENSEÑANZA-APRENDIZAJE}

La enseñanza-aprendizaje de la CO en L2 incluye diversos factores que la hacen adoptar múltiples formas. Por un lado, puede realizarse en entornos presenciales o en entornos a distancia; se pueden utilizar recursos tradicionales (cintas de casete o CD de audio) o novedosos (las TIC); se puede enseñar separada de las demás destrezas o, más comúnmente, como parte de tareas que requieren una interacción entre distintas destrezas; el papel del profesor puede tener diversos niveles de relevancia, así como el papel del alumno puede ser más o menos activo; por último, las tareas pueden presentar múltiples formatos, según el microbjetivo, tiempo disponible, tamaño, nivel del grupo, etc.

De todos estos aspectos que intervienen de un modo u otro en el contexto de la enseñanza de la $\mathrm{CO}$, a continuación se detallan los siguientes: el papel del alumno y el concepto de tarea, en otras palabras, los dos elementos más relevantes del triángulo didáctico (profesor, alumno, tarea).

\subsection{El alumno}

Cuando se habla de la $\mathrm{CO}$, hay que tener en cuenta que en esta destreza entran en juego tres fases, aunque en ciertas situaciones no se pase necesariamente por las tres: reconocimiento, procesamiento y uso del lenguaje (Buck, 2001; Rost, 2002). En la mayoría de las actividades tradicionales de CO en la clase de L2, se ha desarrollado sobre todo la primera fase (reconocimiento, principalmente lingüístico) aunque, afortunadamente, en muchas de las tareas actuales se tienden a trabajar las tres sin distinción.

La fase del procesamiento es la de la construcción del mensaje, a la que ya se ha aludido anteriormente en estas páginas. Aunque la CO (a menudo junto con la comprensión lectora) se ha considerado generalmente como una destreza pasiva o receptiva, no lo es estrictamente hablando, y prueba de ello es que implica un procesamiento y un uso activo de la lengua. En ella, «in order to reconstruct the message that the speaker intends, the hearer must actively contribute knowledge from both linguistic and nonlinguistic sources» (Littlewood, 1981:66). Por este motivo, en lugar de considerar al alumno como una esponja que absorbe información, se le ha de otorgar el papel de actante o agente en el proceso de reconstrucción de significado.

Cabe señalar que, con el objetivo de incrementar el nivel de participación del alumno, es necesario diseñar tareas centradas en él; al fin y al cabo, es el alumno el que ha de realizar el trabajo y desarrollar sus propias destrezas de utilización de la lengua. Así, se le podrá otorgar el grado de control necesario sobre el contenido de la lección o de la tarea. Existen distintos procedimientos que se pueden llevar a cabo para ampliar el protagonismo del alumno en su propio proceso de aprendizaje (Nunan, 1999): personalizar el contenido de modo que los alumnos aporten algo propio a la tarea, diseñar tareas de extensión que utilicen el material como punto de partida y que

\footnotetext{
${ }^{4}$ Por ejemplo, los alumnos pueden ver un extracto de una película y preparar posteriormente preguntas dirigidas al protagonista y relacionadas con la película en cuestión, como si se tratara de una entrevista.
} 
guíen a los alumnos para que aporten parte de contenido ellos mismos, ${ }^{4}$ explicitar a los alumnos los objetivos pedagógicos que se persiguen con la tarea, proporcionar un abanico de opciones junto a una tarea concreta para que así elijan ellos el contenido definitivo de la misma, darles la oportunidad de utilizar sus propios conocimientos y experiencias anteriores en el contexto de la tarea, o fomentar el desarrollo de una actitud reflexiva con respecto al aprendizaje, así como el desarrollo de habilidades de autoevaluación y auto-seguimiento. Estos y otros procedimientos ayudarán a los alumnos a sentirse capaces de utilizar la lengua por sí mismos y de monitorizar su proceso de aprendizaje. Este aumento de su protagonismo influye, además, en la motivación para seguir mejorando y ampliando esa independencia que les lleva a mejorar sus destrezas comunicativas en L2.

Entre los elementos de más relevancia a tener en cuenta al hablar del alumno, se encuentran las estrategias de aprendizaje de las que hacen uso. Estas estrategias han sido estudiadas intensamente en las últimas décadas, tanto en términos pedagógicos generales, como en términos de enseñanza-aprendizaje de la L2, e incluso en términos de estrategias de CO en particular (O'Malley y Chamot, 1990; Oxford, 1990; Goh, 1998; Farrell y Mallard, 2006).

Normalmente, las estrategias de aprendizaje de L2 se dividen en dos clases diferenciadas: las que se utilizan para el aprendizaje a largo plazo, ${ }^{5}$ y las que se adoptan para la situación de aprendizaje en sí, también denominadas estrategias de uso. Éstas últimas se dividen, según Cohen (1998:5-6), en cuatro categorías: (1) estrategias de recuperación (usadas para recuperar material lingüístico almacenado en la memoria), (2) estrategias de ensayo (utilizadas para ensayar estructuras de la lengua meta), (3) estrategias de tapadera (cuyo fin es crear la impresión de que se tiene control sobre el material cuando en realidad es lo contrario) y (4) estrategias de comunicación (centradas en transmitir un mensaje que sea tanto informativo como significativo para el oyente).

En lo que respecta a las estrategias específicas para desarrollar la $\mathrm{CO}$, que ayudan a los alumnos a escuchar eficientemente, las principales son: predecir, inferir, monitorizar, aclarar, responder y evaluar (Rost, 2002). No obstante, la tabla 1, a continuación, presenta una selección más amplia de estrategias concretas de aplicación directa a la destreza de la CO. La autora que las recopila (Oxford, 1990: 317-320) las divide primeramente en 2 grandes grupos: directas (D) e indirectas (I). Dentro de las directas, realiza una diferenciación entre estrategias de memoria $(\mathrm{M})$, cognitivas $(\mathrm{C})$ y de compensación (CC) y dentro de las indirectas, incluye las metacognitivas (M), las afectivas (A) y las sociales (S). ${ }^{6}$

\footnotetext{
${ }^{5}$ Como registrarse en un chat de voz de Internet en la lengua meta o llevar un control de las películas vistas cada mes en forma de fichas y con ejemplos de la lengua aprendida.

${ }^{6}$ Las siglas anteriores se utilizan en la tabla 1 para indicar el grupo al que pertenece cada estrategia. Así, por ejemplo, DC equivale a una estrategia Directa Cognitiva.
} 


\begin{tabular}{|c|c|c|}
\hline AGRUPAR (DM) & $\begin{array}{c}\text { ASOCIAR/ ELABORAR } \\
(\mathrm{DM})\end{array}$ & $\begin{array}{l}\text { UTILIZAR IMÁGENES } \\
\text { (DM) }\end{array}$ \\
\hline $\begin{array}{l}\text { Crear campos semánticos } \\
\text { (DM) }\end{array}$ & $\begin{array}{l}\text { Utilizar palabras clave } \\
(\mathrm{DM})\end{array}$ & $\begin{array}{l}\text { Representar sonidos en la } \\
\text { memoria (DM) }\end{array}$ \\
\hline $\begin{array}{l}\text { Utilizar una respuesta o } \\
\text { sensación física }(\mathrm{DM})\end{array}$ & $\begin{array}{l}\text { Utilizar técnicas mecánicas } \\
\text { (DM) }\end{array}$ & Repetir (DC) \\
\hline $\begin{array}{l}\text { Captar la idea rápidamente } \\
\text { (DC) }\end{array}$ & $\begin{array}{l}\text { Razonar de modo deducti- } \\
\text { vo (DC) }\end{array}$ & Analizar expresiones (DC) \\
\hline $\begin{array}{l}\text { Analizar distintas lenguas } \\
\text { contrastivamente (DC) }\end{array}$ & Traducir (DC) & Transferir (DC) \\
\hline Tomar notas (DC) & Resumir (DC) & $\begin{array}{l}\text { Utilizar claves lingüísticas } \\
\text { o de otro tipo (DCC) }\end{array}$ \\
\hline $\begin{array}{l}\text { Repasar y vincular los co- } \\
\text { nocimientos lingüísticos } \\
\text { previos (IM) }\end{array}$ & Prestar atención (IM) & Organizar (IM) \\
\hline Marcarse objetivos (IM) & $\begin{array}{l}\text { Buscar oportunidades para } \\
\text { practicar (IM) }\end{array}$ & $\begin{array}{l}\text { Identificar los objetivos de } \\
\text { las tareas (IM) }\end{array}$ \\
\hline Auto-evaluarse (IM) & $\begin{array}{l}\text { Realizar auto-seguimiento } \\
\text { (IM) }\end{array}$ & $\begin{array}{l}\text { Respirar, relajarse y reca- } \\
\text { pacitar (IA) }\end{array}$ \\
\hline Utilizar música (IA) & Usar el humor (IA) & $\begin{array}{l}\text { Realizar enunciados positi- } \\
\text { vos (IA) }\end{array}$ \\
\hline $\begin{array}{l}\text { Arriesgarse (con pruden- } \\
\text { cia) (IA) }\end{array}$ & $\begin{array}{l}\text { Recompensarse a uno mis- } \\
\text { mo (IA) }\end{array}$ & $\begin{array}{l}\text { Escribir un diario de } \\
\text { aprendizaje (IA) }\end{array}$ \\
\hline $\begin{array}{l}\text { Preguntar para comprobar } \\
\text { o esclarecer (IS) }\end{array}$ & $\begin{array}{l}\text { Cooperar con compañeros } \\
\text { (IS) }\end{array}$ & $\begin{array}{l}\text { Desarrollar un entendi- } \\
\text { miento cultural (IS) }\end{array}$ \\
\hline
\end{tabular}

Fomentar el desarrollo de las estrategias de aprendizaje en L2 es muy importante, pero hay que tener precaución y no obcecarse en desarrollar todas las estrategias a toda costa, especialmente teniendo en cuenta el gran número de estrategias de aprendizaje que han ido surgiendo en las últimas décadas divididas en diversas categorías y subcategorías establecidas por diversos autores. Como explica Rost (2002:111), hay que centrarse en las adecuadas según el alumno y la situación:

The point of incorporating strategy instruction into the language teaching is not to «accomplish» as many strategies as possible, but rather to focus the learners on particular cognitive plans they can employ to help them overcome obstacles in language use or language learning.

Es cierto que es importante desarrollar estrategias de aprendizaje, pero las necesarias en cada caso (independientemente de cómo las llame uno u otro autor), y las que vayan a ayudar verdaderamente a los alumnos en su proceso de aprendizaje de la L2.

\subsection{Las tareas}

Resulta necesario realizar actividades de $\mathrm{CO}$ alrededor de una tarea concreta, para que los alumnos interactúen de forma organizada según lo que escuchan y comprueben de un modo activo, y por ellos mismos, lo que han comprendido (Ur, 1984: 
25). En este apartado se explica el concepto de tarea de CO, los beneficios del uso de la misma, sus tipos principales y su estructura básica.

En nuestra vida diaria, cuando escuchamos algo, tenemos normalmente un objetivo específico para hacerlo. En clase, este objetivo es por lo general sólo lingüístico $\mathrm{y}$, por este motivo, se debe integrar un objetivo no-lingüístico, en forma de tarea, con el fin de que el alumno se sienta motivado para escuchar y el tipo de comprensión se pueda transportar a situaciones de la vida real. Una definición simple y concisa de tarea (didáctica) en lo que respecta a la CO podría ser la siguiente: «un acto diseñado para proporcionar un propósito a la escucha». De este modo, el alumno puede saber qué tipo de información ha de esperar y cómo debe o puede reaccionar ante ella.

Antes de continuar con la exposición del concepto de tarea, cabe explicar los tres tipos de escucha que pueden existir según el objetivo perseguido; Rost (2002:137) señala tres tipos posibles de actividades de CO: (1) escucha intensiva, (2) escucha selectiva y (3) escucha interactiva. En la escucha intensiva, se pretende distinguir sonidos, palabras, frases y/o unidades gramaticales o pragmáticas concretas, y esto se lleva a cabo por medio de dictados, ejercicios de rellenar huecos (cloze), de identificación de errores u otras variaciones relacionadas; este tipo de escucha está directamente relacionada con la dimensión receptiva de la CO. En la escucha selectiva, el alumno ha de centrar su atención en información específica, en lugar de comprender y recordar todo lo que oye; esta escucha suele estar basada en actividades de toma de notas, según los requisitos específicos de la tarea y está relacionada con la dimensión constructiva de esta destreza. Finalmente, en la escucha interactiva, se realiza una escucha como parte de una conversación donde exista interacción y negociación; se trata de una tarea comunicativa que posee un objetivo tangible de interacción y que se relaciona con la dimensión negociadora de la $\mathrm{CO}$ en concreto.

Independientemente del tipo de escucha, la tarea ofrece a los alumnos de L2 la oportunidad de aportar una respuesta inmediata a aquello que se ha escuchado, que es lo que requieren, por regla general, las situaciones de la vida real. Aunque la realidad de la clase no permita normalmente reproducir el mismo tipo de respuestas que se dan en la realidad, lo importante es que exista una respuesta, aunque ésta sea silenciosa (física, o por escrito). Otra ventaja de organizar una actividad alrededor de una tarea es que resulta más motivador e interesante para el alumno responder de modo activo a un texto oral que escucharlo pasivamente. Una tarea bien diseñada tiene, además, elementos lúdicos y de resolución de problemas que aumentan la efectividad del aprendizaje. Aunque no es estrictamente necesario graduar el nivel de la tarea para que haya un progreso efectivo en la $\mathrm{CO}$, es conveniente que las tareas en el aula se adapten al conocimiento del idioma general del alumno. Estas tareas deben complementarse fuera del aula con exposición a input más o menos indiscriminado, dado que se suele tratar de pocas horas a la semana.

Las tareas pueden adquirir diversas formas. Pueden ser abiertas o cerradas (Rost, 2002); un ejemplo de tarea abierta es resumir las ideas principales de un texto y uno de tarea cerrada, completar los huecos con información textual que se ha de obtener a partir de la escucha. Las tareas también se pueden distinguir de acuerdo con (1) el papel del alumno, (2) las estrategias requeridas y (3) el énfasis principal (Nunan, 1999): 
1. Desde el punto de vista del alumno, se pueden distinguir tareas recíprocas (en forma de diálogo, con o sin participación del alumno) y no recíprocas (en forma de monólogos, tales como anuncios en el transporte público, llamadas telefónicas, charlas o conferencias). Ambos tipos reproducen situaciones para las que el sujeto debe estar preparado, aunque posiblemente las primeras sean más funcionales (por su componente interactivo) y debieran recibir una atención mayor.

2. Según las estrategias requeridas, se puede hablar de tareas para captar la idea principal, obtener información específica, realizar inferencias a partir de lo escuchado, realizar distinciones fonéticas, etc. A pesar de que la primera tarea de este grupo es una de las actividades más comunes de la escucha, es importante practicar también las demás porque su manejo es necesario para comprender un importante número de actividades cotidianas.

3. En términos del énfasis principal de la tarea, las hay que se centran en elementos lingüísticos (aumentar o afianzar conocimientos gramaticales, fonológicos, léxicos, etc.) y las que se centran en el contenido (semántico, pragmático y cultural). Ambos tipos son relevantes en el entorno educativo, pero la segunda de ellas resulta considerablemente más práctica para transferirla a situaciones comunicativas reales fuera del entorno de la clase.

Las tareas de $\mathrm{CO}$, independientemente de su tipo, se estructuran, por regla general, en tres fases: pre-escucha, escucha y post-escucha (pre-listening, while-listening, post-listening).

El objetivo principal de la fase «pre-escucha» es crear expectativas acerca de lo que se va a escuchar más adelante. Se permite así a los alumnos activar los esquemas mentales de conocimiento pertinentes y los conocimientos previos necesarios que les permitirán estar preparados para comprender mejor, posicionando sus ideas en el contexto adecuado. El porcentaje de tiempo que se suele dedicar a esta fase es el $15 \%$ del total de la tarea.

Las actividades de la fase «escucha» tienen como objetivo mantener la atención del alumno durante todo el proceso. Otra de sus funciones es permitirle relacionar el texto escuchado con las expectativas surgidas en la fase anterior. Para que la tarea se lleve a cabo con éxito, estas actividades han de ser interesantes con el fin de que hagan a los alumnos querer escuchar el texto. Además, es importante que el nivel de dificultad sea razonable para que se pueda reforzar la comprensión sin tener que desviar la atención a demasiados elementos lingüísticos nuevos que puedan hacer fracasar la tarea. Con dicho fin, el profesor puede ajustar el nivel aportando u ocultando elementos de apoyo a la comprensión. Por último, la forma de la actividad debe ser lo más simple posible, para que el alumno pueda escuchar el texto y no tenga que concentrarse demasiado en comprender lo que hay que hacer. Esta fase ocupa el $50 \%$ de la tarea, aproximadamente.

La última fase incluye actividades «post-escucha». Se realizan al término de la escucha y, aunque pueden estar o no relacionadas con las actividades de las dos fases anteriores, es preferible que lo estén. Entre los propósitos de esta fase destaca el comprobar si los alumnos han entendido lo que debían y si han realizado con éxito las actividades de las dos fases anteriores. Otra función es reflexionar acerca de los po- 
sibles problemas de comprensión surgidos en todo el proceso de la tarea. Es por eso que en esta fase se tiende a trabajar sobre el tema y la lengua encontrados en el texto, para poder transferir este aprendizaje a otros contextos o destrezas, cumpliendo así la dimensión metacognitiva que ha de satisfacer toda actividad de CO. Así, para poder afianzar y reforzar el éxito de la tarea, es necesario que se incluya aquí una respuesta inmediata por parte del profesor, en la que se resalten todos los elementos positivos surgidos, sin olvidar una exposición de los problemas más relevantes que hayan podido aparecer (Ur, 1984). Finalmente, esta fase, que ocupa aproximadamente el $35 \%$ de la tarea, tiene que ser lo suficientemente motivadora y original para que consiga mantener (a estas alturas de la tarea) la atención de los alumnos.

En la tabla siguiente se proporcionan ejemplos de actividades para las distintas fases .

Tabla 2. Ejemplos de actividades de las distintas fases de la tarea (Underwood, 1990).

\begin{tabular}{|l|l|l|}
\hline \multicolumn{1}{|c|}{ "Pre-escucha" } & \multicolumn{1}{|c|}{ "Escucha" } & \multicolumn{1}{c|}{ "Post-escucha" } \\
\hline $\begin{array}{l}\text { Proporcionar información de } \\
\text { fondo sobre el tema a tratar } \\
\text { por parte del profesor }\end{array}$ & Señalar objetos en imágenes & $\begin{array}{l}\text { Role-plays (actividades de ex- } \\
\text { presión oral que tienden a imi- } \\
\text { tar situaciones observadas en } \\
\text { el texto oral) }\end{array}$ \\
\hline $\begin{array}{l}\text { Observar y comentar imáge- } \\
\text { nes relacionadas }\end{array}$ & $\begin{array}{l}\text { Ordenar imágenes de acuerdo } \\
\text { con la historia }\end{array}$ & $\begin{array}{l}\text { Ejercicios de resolución de } \\
\text { problemas o de toma de deci- } \\
\text { siones }\end{array}$ \\
\hline Preguntas y respuestas orales & $\begin{array}{l}\text { Completar tablas o formula- } \\
\text { rios }\end{array}$ & $\begin{array}{l}\text { Resúmenes (a partir de notas y } \\
\text { de memoria) }\end{array}$ \\
\hline $\begin{array}{l}\text { Formular preguntas que se } \\
\text { podrán responder posterior- } \\
\text { mente, imaginando ahora las } \\
\text { respuestas }\end{array}$ & $\begin{array}{l}\text { Responder a preguntas de ver- } \\
\text { dadero o falso o de respuesta } \\
\text { múltiple diseñadas de un } \\
\text { modo simple y bien estructu- } \\
\text { rado }\end{array}$ & $\begin{array}{l}\text { Identificar relaciones entre los } \\
\text { distintos hablantes, sus actitu- } \\
\text { des o estado de ánimo }\end{array}$ \\
\hline $\begin{array}{l}\text { Llevar a cabo un debate previo } \\
\text { sobre el tema de la escucha }\end{array}$ & $\begin{array}{l}\text { Reconocer errores en el texto } \\
\text { (reproducido por escrito) }\end{array}$ & $\begin{array}{l}\text { Escribir una redacción o diálo- } \\
\text { go a partir del contenido de la } \\
\text { escucha }\end{array}$ \\
\hline
\end{tabular}

Obviamente, cada actividad presenta una serie de ventajas e inconvenientes que se deben valorar para cada situación de aprendizaje; por ejemplo, las actividades de verdadero/falso, más mecánicas y simples, tienen la ventaja de ser rápidas y de poder aplicarse a discursos complejos. En cualquier caso, un aspecto que no se ha de perder nunca de vista es el éxito de la tarea: «tasks should be success-oriented. This not only improves motivation [...] but also improves the effectiveness of the listening practice given» (Ur, 1984:27). Completar una tarea con éxito, sea del tipo que sea e independientemente de las actividades que contenga, provocará un aumento en la motivación que tendrá una influencia directa en la mejora de la eficacia de la práctica de CO llevada a cabo. 


\section{LA eVALUACión DE LA CO}

Resulta muy común confundir la enseñanza de la $\mathrm{CO}$ con su evaluación, dado que las formas más comunes de práctica de esta destreza han consistido durante muchos años en una simple evaluación de la misma. Según Anderson y Lynch (1988:66) «the difficulty is that the classroom methods that most teachers think of as tools for teaching listening comprehension in fact amount to a form of continuous testing». Este hecho es negativo porque los alumnos, por un lado, no trabajan para mejorar las estrategias necesarias para comprender mejor, y por otro, no se sienten motivados para realizar una actividad que siempre lleva implícito el elemento evaluador de sus capacidades receptivas.

Cohen (1980:1) habla de las «wh-questions of classroon testing»: por qué evaluar, cuándo evaluar, qué evaluar, para qué evaluar y cómo evaluar. Las razones para evaluar (el «por qué») pueden ser múltiples, desde conocer los niveles del alumnado para formar grupos hasta otorgar un título académico. En cuanto al momento (el «cuándo»), este aspecto es muy relativo; no obstante, se tiende a evaluar siempre al principio y al final de cada curso, así como de modo continuo (cada cierto periodo de tiempo). Cuando se plantea la pregunta de «qué» se quiere evaluar, es necesario definir el constructo, elemento abstracto que contiene tanto el enfoque teórico utilizado, como los textos seleccionados y las tareas a realizar (Buck, 2001). En términos de «para qué» evaluar, la mayoría de las pruebas de $\mathrm{CO}$ utilizadas por los profesores son pruebas de logros que evalúan el grado de dominio de lo enseñado. Otro tipo de pruebas relativamente comunes son las pruebas de nivel (anteriores o posteriores al proceso de aprendizaje), diseñadas para comparar el nivel de unos alumnos frente a otros (Rost, 2002). El «cómo», por último, se refiere a la forma y al contenido de las pruebas de evaluación en sí.

Según Rost (1990:175), «from a practical perspective, testing is a critical area in language education as results of testing often influence the future of learners, the professional evaluation of instructors, and the direction of curriculum design». En otras palabras, la evaluación en el ámbito educativo siempre resulta crucial, por las múltiples consecuencias que se pueden derivar de sus resultados. Habida cuenta de ello, cabe señalar que la evaluación de la CO propiamente dicha resulta normalmente más complicada, lleva más tiempo y es más difícil de poner en práctica que la evaluación de otras destrezas como, por ejemplo, la comprensión lectora (Buck, 2001).

El presente apartado se centra en explicar los aspectos más significativos del «qué» y el «cómo» evaluar la $\mathrm{CO}$, ya que el resto de preguntas dependen del contexto concreto de evaluación. Por tanto, a continuación se analizan los enfoques principales de evaluación de la $\mathrm{CO}$ y los elementos principales de las pruebas.

\subsection{Los enfoques de evaluación}

Históricamente, se han distinguido tres enfoques principales para la evaluación de la CO en L2: diferenciador, integrado y comunicativo (Buck, 2001:61). Estos enfoques no constituyen conjuntos de ideas cerradas y precisas, sino que se centran en qué aspectos concretos de la lengua se tienen en cuenta a la hora de evaluar. 
El enfoque diferenciador se asocia a teorías conductistas y se centra en la identificación de elementos lingüísticos de un modo diferenciado y aislado, así como en la evaluación de las distintas destrezas por separado (Oller, 1979). Se trata, por ejemplo, de evaluar el grado en el que el alumno es capaz de distinguir ciertas entonaciones, estructuras gramaticales, fonemas o elementos léxicos concretos. Las pruebas que se asocian a este enfoque suelen tener respuestas cerradas previamente seleccionadas, de tipo verdadero-falso o preguntas de respuesta múltiple. Los aspectos teóricos fundamentales en los que se basan estas ideas son: la importancia de aislar los diferentes elementos dentro del flujo oral y el hecho de que se considera la lengua hablada prácticamente igual que la escrita, por lo que hay que saber distinguir sus elementos de un modo análogo. Obviamente, estas ideas se consideran limitadas hoy en día (Buck, 2001). ${ }^{7}$ No obstante, lo positivo del enfoque diferenciador es que, al obligar al alumno a centrar su atención en la comprensión de un elemento determinado, éste se ve obligado a procesar e interpretar otros elementos.

El enfoque integrado parte de la idea de que existen relaciones importantes entre los diferentes componentes del lenguaje, por lo que es necesario saber en qué modo se relacionan estos elementos. Este enfoque surge a partir de distintos estudios de la década de 1970 que empiezan a centrarse en el «uso» de la lengua, en lugar de en el «conocimiento» de la misma. Oller (1979), uno de los exponentes más importantes de esta teoría (que él prefiere llamar enfoque pragmático), reclama la importancia de que «el todo» (en términos lingüísticos) es más importante y mayor que la suma de sus partes. Según este autor, en este tipo de enfoques, las secuencias lingüísticas han de estar relacionadas con contextos extralingüísticos de modo significativo. Los tipos de pruebas están relacionadas aquí en gran medida con predicciones, y también incluyen ejercicios de rellenar huecos, dictados, tareas de repetición de frases, traducción y resumen, etc.

Aunque en el enfoque integrado aún no se menciona la importancia de la comunicación, muestra una diferencia fundamental con el enfoque anterior: mientras el diferenciador evalúa el conocimiento de los componentes de la lengua, el integrado valora el procesamiento de la misma y cómo el conocimiento de las relaciones existentes entre los distintos elementos puede ayudar a realizar predicciones sobre el contenido total del continuo lingüístico. En este sentido, en los tests integrados, tiene especial importancia la llamada «redundancia reducida» de la lengua (Buck, 2001), que se da a distintos niveles (sintáctico, morfológico, fonológico, etc.), y que ayuda a crear expectativas acerca de la misma. Por ejemplo, en he came home last month, el tiempo pasado (came) crea la expectativa de la existencia de un componente adverbial de tiempo, como el que aparece a continuación (last month). Estas ideas se basan en la llamada «gramática de esperanza pragmática» (pragmatic expectancy grammar) de Oller (1979), según la cual el lenguaje siempre crea, en cada situación, un contexto lingüístico determinado que lo acompaña y se va ampliando con cada elemento lingüístico que aparece en el flujo del habla; las expectativas creadas por dichos elementos ayudan a comprender mejor los siguientes conceptos y expresiones que van surgiendo en cada situación.

\footnotetext{
${ }^{7} \mathrm{Al}$ observar la comprensión del lenguaje a nivel local y literal, este enfoque no contempla la redundancia lingüística ni que el procesamiento de cualquier elemento de la lengua lleva consigo una serie de inferencias e interpretaciones de todo el flujo lingüístico escuchado.
} 
$\mathrm{El}$ aspecto negativo de este enfoque es que busca analizar el procesamiento del mensaje de un modo aislado, sin tener en cuenta el contexto situacional, elemento fundamental para comprender la lengua en términos comunicativos. ${ }^{8}$ No obstante, lo positivo es que la noción teórica básica que subyace es el procesamiento del lenguaje a tiempo real, un tipo de procesamiento que ayuda a comprender tanto el significado literal, como el semántico (Buck, 2001).

El último enfoque, el comunicativo, se centra en la comprensión del mensaje, relacionándolo con el contexto comunicativo al que pertenece. Surge, por tanto, a partir de las teorías didácticas de base comunicativa nacidas a finales de 1980. Busca, como el enfoque integrado, evaluar el uso de la lengua; pero, en este caso, hace hincapié en la importancia de comprender dicho uso en una situación determinada y con unos objetivos específicos. Se trata, por tanto, de comprender el uso de la lengua en situaciones reales, contextualizadas, con objetivos de ejecución de un acto determinado y con características auténticas e impredecibles. Las pruebas relacionadas con este enfoque tienden a utilizar textos auténticos o semiauténticos y poseen un propósito comunicativo para la escucha. Según Weir (2005:98), «to test listening we must understand the processing that takes place in real life situations and attempt to see that communication in our tests is anchored in the real world as far as possible». Por tanto, las tareas de evaluación han de ser lo más auténticas y activas posibles, adoptando tantas formas como escenarios comunicativos hay en la realidad.

La noción teórica básica alrededor de la cual se articula este enfoque es que la evaluación de la CO ha de tener en cuenta la comprensión de una situación comunicativa real en su totalidad. Desafortunadamente, la búsqueda de la autenticidad, tanto situacional como interactiva, no siempre es fácil dentro del contexto educativo. De hecho, este aspecto origina uno de los problemas principales de este enfoque: al existir tantas situaciones comunicativas diferentes y tantos temas de conversación, la generalización para aplicar los conocimientos adquiridos a situaciones similares deviene más difícil cuanto más comunicativa sea la prueba. Un inconveniente secundario que acarrea este hecho son las distintas interpretaciones subjetivas a las que puede dar lugar cada situación de acuerdo con la subjetividad de cada oyente. En palabras de Buck (2001: 85):

The basic problem with communicative testing is that language use varies considerably from one individual to another, and one situation to the next [...] Despite the theoretical difficulty of determining what a communicative test is, and the practical difficulties of doing it, communicative testing has had a very powerful and pervasive influence on language testing for more than two decades.

Por lo tanto, a pesar de estos inconvenientes, la influencia de este enfoque dentro de la didáctica de lenguas ha sido muy fuerte durante las últimas décadas. Entre otras razones, esto se debe al énfasis otorgado a la utilización de la lengua de modo comunicativo global en todas las destrezas y en todos los aspectos de la enseñanza-aprendizaje, incluida la evaluación.

\footnotetext{
${ }^{8}$ Se trata más de una comprensión a nivel de la frase, y no exige al alumno realizar inferencias de significados a nivel global del texto, ni relacionar la información lingüística del mensaje con el contexto.
} 
Sea cual sea el enfoque elegido para evaluar, dependiendo de la situación educativa, lo que hay que tener siempre presente es la elección y preparación previa del mismo, así como la validez, fiabilidad y viabilidad de la evaluación en conjunto.

\subsection{Las pruebas de evaluación}

Rost (2002:172-173) clasifica las prácticas de evaluación de la CO más comunes en la actualidad en seis grupos: (1) pruebas de diferenciación de elementos, (2) pruebas integradas, (3) pruebas comunicativas, (4) pruebas de entrevista, (5) pruebas de autoevaluación y (6) la evaluación del portafolio.

1. El primer grupo de pruebas, pruebas de diferenciación de elementos, incluye preguntas de respuesta múltiple, preguntas abiertas acerca del texto oral y pruebas estándar del tipo TOEFL (Test of English as a Foreign Language) o DIALANG.

2. Las pruebas integradas, por su parte, contienen resúmenes abiertos sobre un texto oral, actividades de rellenar huecos en un resumen escrito del texto escuchado y dictados completos o parciales.

3. Las pruebas comunicativas engloban tareas de comunicación escrita y tareas orales y no verbales, relacionadas todas ellas con la escucha de un texto oral. Un ejemplo escrito podría ser escribir un email de solicitud de información después de haber escuchado el anuncio de un hotel, mientras que uno oral podría consistir en seguir indicaciones en un mapa.

4. Las pruebas de entrevista incluyen representaciones (role-plays) con otro alumno o con el profesor y entrevistas orales de mayor duración.

5. El penúltimo grupo, el de la autoevaluación, contiene cuestionarios de evaluación personal o evaluación detallada de la propia destreza, en forma de apuntes periódicos orales o escritos.

6. Por último, la evaluación del portafolio engloba evaluaciones y observaciones periódicas del profesor, tanto objetivas como subjetivas, del proceder de los alumnos en las tareas de clase.

Cabe señalar que las pruebas de evaluación de CO deben tener validez en sí mismas (lo que Weir [2005:11] llama test validity). Hay una serie de criterios de validez que son necesarios para que cualquier prueba o test pueda ser analizado objetivamente y para que sus resultados sean legítimos. Por un lado, cabe destacar la validez teórica, que es la necesidad de definir previamente, y del modo más claro posible, el constructo en su totalidad. Los otros criterios de validez que hay que tener en cuenta son: la validez del contexto (en términos de la representatividad de la tareas y el uso de elementos auténticos y situaciones lo más reales posibles), la validez externa (comparaciones entre criterios estándar o posibles consecuencias que dichas pruebas pueden acarrear) y la validez de las escalas de puntuación (que sean objetivas, fiables, que estén justificadas y que tengan consistencia interna).

Todos estos criterios han de valorarse a la hora de llevar a cabo la prueba o pruebas que se pretendan poner en práctica, aunque algunos tengan que considerarse antes (validez teórica y de contexto) y otros después (validez externa y de puntuación) de la realización de las mismas. Aunque todos los criterios poseen una importancia similar, el último de ellos, el modo de puntuar o valorar las distintas pruebas, es uno de los más injustamente desatendidos. La puntuación depende de los objetivos de las pruebas y de otras circunstancias rela- 
cionadas, y deberá estar directamente relacionada con los contenidos estudiados. Por último, nunca puede olvidarse que el modo de puntuar tiene que estar perfectamente claro y plantearse con el mayor grado posible de objetividad antes de ponerlo en práctica. En palabras de Cohen (1980:34):

Scoring is often taken for granted. The bulk of the concern is given to eliciting the test data. Less attention is paid to determining the number of points that each item or procedure is to receive, and even less attention is paid to determining the value of the score.

It is suggested in the literature that teachers develop a scoring key precisely in order to avoid the «I have a general idea of what I'm looking for» approach.

En definitiva, la puntuación que se otorga a una prueba de evaluación (así como el porcentaje de la misma que se obtiene en cada una de sus secciones, en su caso) requiere una atención igual o mayor que la de la búsqueda de los diferentes elementos que conforman dicha prueba.

Probablemente, lo más relevante dentro del proceso de evaluación de la CO es tener en cuenta que «the safest approach for teachers is to try and make test tasks approximate as closely as possible to the real life abilities they wish to say something about, in terms of both situational and interactional authenticity» (Weir, 2005:102). Cuanto más auténticas y comunicativas sean, mejor valorarán la competencia real del alumno.

El presente artículo ha pretendido esclarecer las claves para comprender la destreza de la $\mathrm{CO}$ en términos de su definición, los elementos que intervienen en el proceso constructivo, los aspectos más relevantes de su enseñanza-aprendizaje, y los principales enfoques y métodos de evaluación A modo de conclusión, la figura 1 presenta un mapa conceptual de los principales elementos que caracterizan esta destreza, siguiendo las explicaciones aportadas a lo largo de estas páginas.

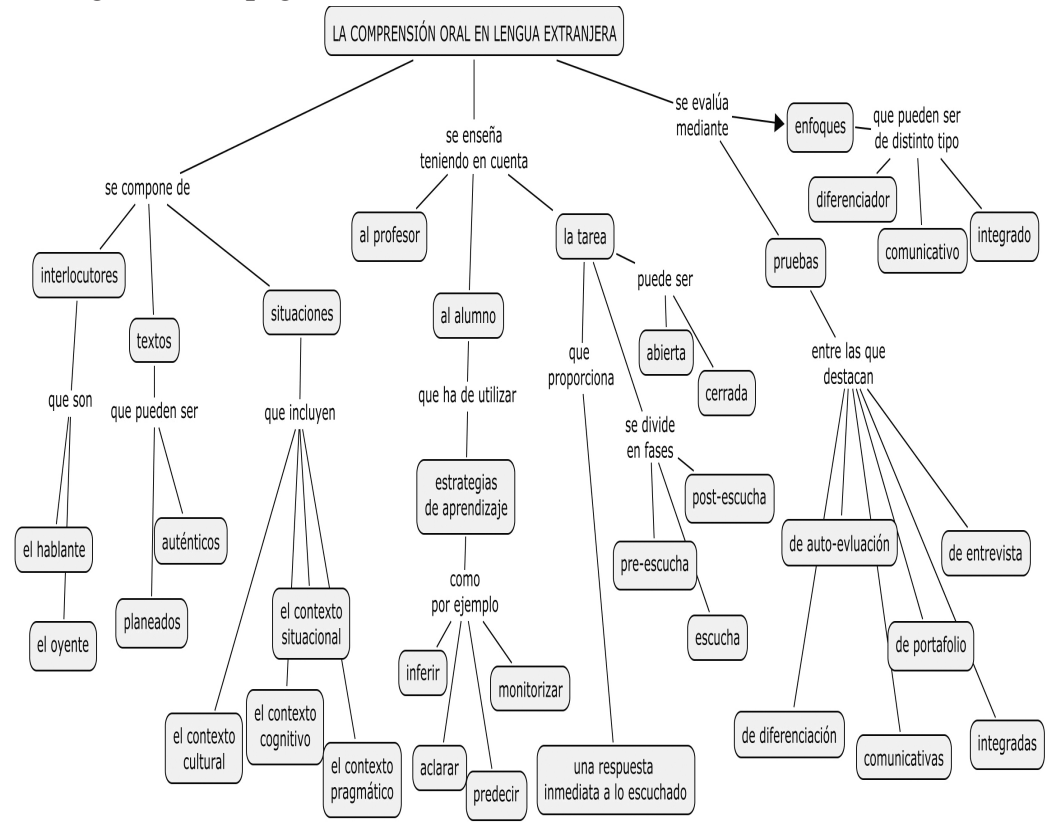

Fig. 1. Resumen conceptual de la comprensión oral. 


\section{REFERENCIAS:}

ANDERSON, Anne y LYNCH, Tony (1988): Listening. Nueva York, Oxford University Press.

Austin, John Langshaw (1962): How to Do Things with Words. The William James Lectures Delivered at Harvard University in 1955, Oxford, Oxford University Press.

BACON, S. M. (1992): «Authentic listening in Spanish: how learners adjust their strategies to the difficulty of the input», Hispania, 75(2), 398-412.

Brown, Gillian y Yule, George (1983): Teaching the Spoken Language, Cambridge, Cambridge University Press.

BRown, Gillian (1995): Speakers, Listeners and Communication, New York, Cambridge University Press.

BucK, Gary (2001): Assessing Listening, Cambridge, Cambridge University Press.

CoHEn, Andrew D. (1980): Testing Language Ability in the Classroom, Rowley, MA, Newbury House Publishers.

CoHEn, Andrew D. (1998): Strategies in Learning and Using a Second Language. Nueva York, Longman.

CORNAIRE, Claudette (1998): La Compréhension Orale, Paris, CLE International.

De Bot, Kees, LowIE, Wander y VersPoOr, Marjolijn (2005): Second Language Acquisition: An Advanced Resource Book, New York, Routledge.

FARRELL, T. S. Y MALLARD, C. (2006): «The use of reception strategies by learners of French as a foreign language», The Modern Language Journal, 90(3), 338-352.

GoH, C. C. (1998): «How ESL learners with different listening abilities use comprehension strategies and tactics», Language Teaching Research, 2(2), 124-147.

Gremmo, M. y Holec, H. (1990): «La compréhension orale: Un processus et un comportement», en GaOnach, D., Mac Nally, D. y Ballaire, M. F., eds, Acquisition d'une langue étrangère, l'approche cognitive, Paris, Hachette, 30-40.

GrICE, H. P. (1969): «Utterer's meaning and intention», The Philosophical Review, 78(2), 147-177. HALLIDAY, Michael (1989): Spoken and Written Language, Oxford, Oxford University Press.

HERRON, C. (1994): An investigation of the effectiveness of using an advance organizer to introduce video in the foreign language classroom, The Modern Language Journal, 78(2), 190-198.

Krashen, Stephen y TerRell, Tracy (1983): The Natural Approach: Language Acquisition in the Classroom, Oxford, Pergamon Press.

LADO, Robert (1957): Linguistics across Cultures, Ann Arbor, Michigan, University of Michigan Press.

LitTlewood, William (1981): Communicative Language Teaching. An Introduction, New York, Cambridge University Press.

LONG, D. R. (1990): «What you don't know can't help you: An exploratory study of background knowledge and second language listening comprehension», Studies in Second Language Acquisition, 12(1), 65-80.

Lynch, Tony (1996): Communication in the Language Classroom, New York, Oxford University Press.

MiLLER, L. (2003): «Developing listening skills with authentic materials», ESL Magazine, 6(2), 16-18.

NunAn, David (1999): Second Language Teaching and Learning, Florencia, Kentuky, Heinle \& Heinle Publishers.

Oller, John W. (1979): Language Tests at School, Essex, Longman.

O'MaLley, J. Michael. у Снамот, Anna Uhl (1990): Learning Strategies in Second Language Acquisition, Cambridge, Cambridge University Press.

OXFORD, Rachel (1990): Language Learning Strategies: What Every Teacher should Know, Boston, Heinle \& Heinle. 
Peterson, E. Y COLTRANE, B. (2003): «Culture in second language teaching», CAL Digest, (EDO-FL-03-

09), Centre for Applied Linguistics. www.cal.org/resources/digest/0309peterson.html

Rost, Michael (1990): Listening in Language Learning, New York, Longman.

Rost, Michael (2002): Teaching and Researching Listening, London, Longman.

SEARLE, John (1969): Speech Acts, Cambridge, Cambridge University Press.

SPERBER, Dan y WiLSON, Deirdre (1986): Relevance: Communication and Cognition, Oxford, Basil Blackwell.

UNDERWOOD, Mary (1990): Teaching Listening, London, Longman.

UR, Penny (1984): Teaching Listening Comprehension, Cambridge, Cambridge University Press.

WeIR, Cyril (2005): Language Testing and Validation: An Evidence-based Approach, New York, Palgrave Macmillan. 